\title{
LOS MUSGOS DE LA SIERRA DE ALCAPARROSA, MEXICO
}

\author{
Angeles Cardenas S. y Claudio Delgadillo M. \\ Instituto de Biología, UNAM \\ Apartado Postal 70-233 \\ 04510 México, D.F.
}

\begin{abstract}
RESUMEN
Se registran 84 especies y variedades de musgos de la Sierra de Alcaparrosa. Las familias mejor representadas son las Pottiaceae (26 especies) y las Bryaceae (10 especies); las demás familias incluyen de 1 a 4 especies en el área. También están presentes 7 especies de musgos cleistocárpicos.

Entre los seis elementos fitogeográficos, el más numeroso es el de amplia distribución. Son también importantes el elemento endémico (sólo conocido de la República Mexicana), el elemento disyunto (con distribución discontinua entre México y Sudamérica; dos de sus especies están representadas en Africa y Australia) y el elemento mesoamericano (distribuido desde México hasta el norte de Sudamérica).
\end{abstract}

\begin{abstract}
The known moss flora of Sierra de Alcaparrosa consists of 84 species and varieties. The Pottiaceae (26 species) and the Bryaceae (10 species) are the families best represented; the remaining include between 1 and 4 species in the area. The moss flora includes a group of 7 cleistocarpic mosses.

Among the six phytogeographical elements that of wide distribution is the largest. Other important elements in this moss flora are the endemic (known only from Mexico), disjunct (between Mexico and South America; two species are represented in Africa and Australia) and the mesoamerican (distributed from Mexico to northern South America).
\end{abstract}

\section{INTRODUCCION}

Como parte de ciertos estudios florísticos y fitogeográficos en el Eje Neovolcánico (cf. Delgadillo, 1985; 1987a; 1988), en 1982 se iniciaron los trabajos para la preparación de un catálogo de los musgos del Valle de México. Estos últimos han dado lugar a la publicación de algunos trabajos (Cárdenas, 1987; 1988; 1989) en los que se describen brevemente los rasgos sobresalientes del Valle de México y se enlistan registros briológicos nuevos para la zona o para el país.

Desde el punto de vista briológico, el Valle de México es una de las regiones mejor conocidas del país. Sin embargo, su porción septentrional ha recibido poca atención; entre los trabajos que hacen referencia directa a ésta se encuentran el de Crum (1951) que registra 32 especies de musgos para la Sierra de Pachuca y el de Alfaro y Castillo (1986) en el que se estudia la distribución por tipos de vegetación y se enlistan 169 especies y variedades de musgos para la Sierra de Pachuca. 
Para aportar nuevos datos sobre la flora briológica de la mitad septentrional del Valle de México, en esta contribución presentamos una lista de los musgos recolectados en la Sierra de Alcaparrosa y agregamos comentarios sobre su distribución geográfica.

\section{EL AREA DE ESTUDIO}

La mitad septentrional del Valle de México está situada al norte de los $19^{\circ} 37^{\prime} \mathrm{N}$ (Fig. 1) y comprende unos $3500 \mathrm{~km}^{2}$ ocupados por una llanura y varios macizos

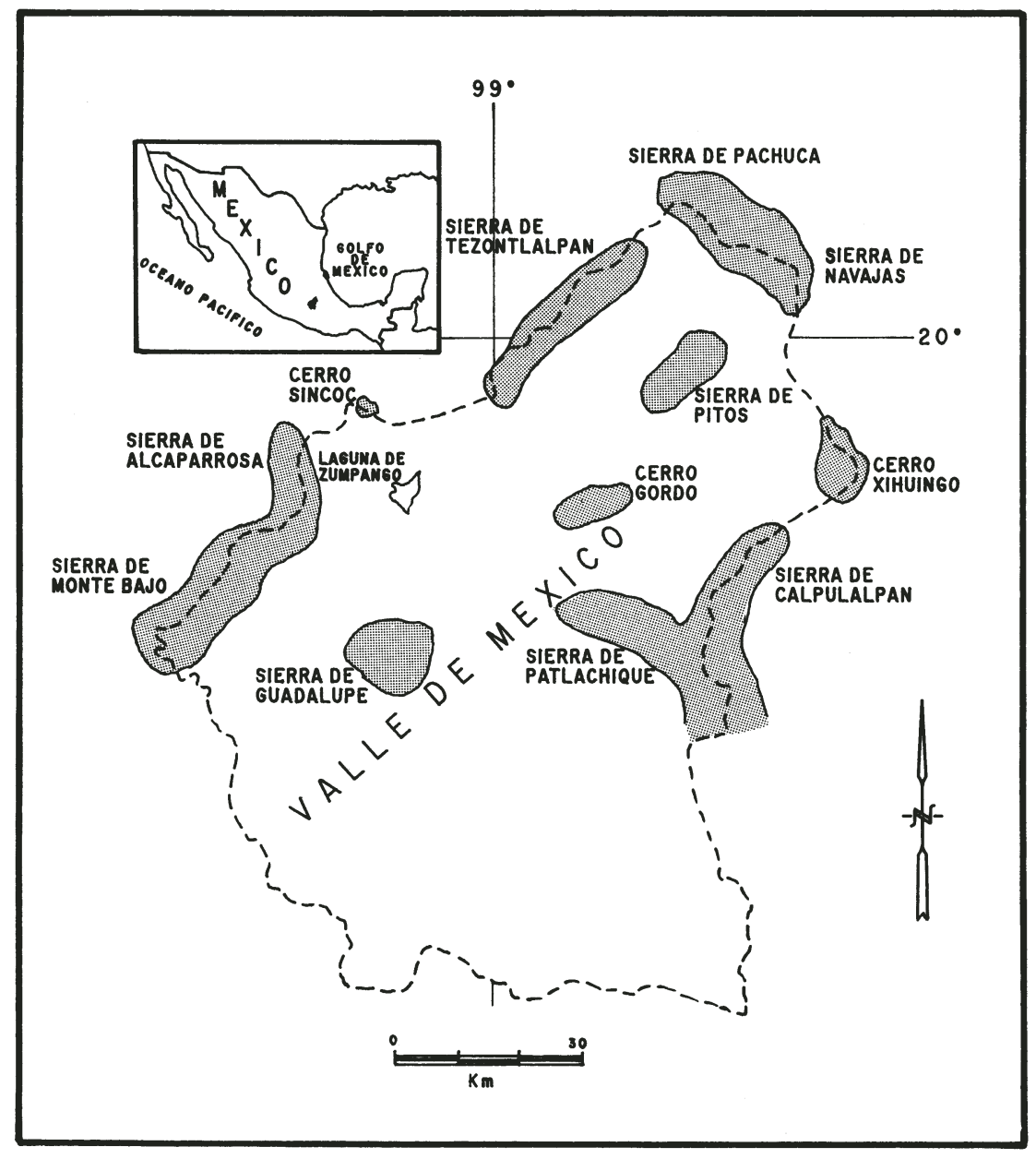

Fig. 1. La Sierra de Alcaparrosa se localiza en la mitad septentrional del Valle de México. Redibujado de Rzedowski et al. (1964). 
montañosos entre los que destacan, por su altura, las serranías de Monte Bajo y la de Alcaparrosa en el suroeste. Hacia el norte se encuentra la Sierra de Pachuca; en la parte oriental sobresale el Cerro Xihuingo y los cerros del Maguey, del Cuello y Colorado que forman parte de la Sierra de Calpulalpan; aislados en medio de la llanura central se encuentran la Sierra de los Pitos y el Cerro Gordo (Rzedowski et al., 1964).

La Sierra de Alcaparrosa está situada a los $19^{\circ} 41^{\prime} \mathrm{N}$ y $99^{\circ} 20^{\prime} \mathrm{W}$ (Núñez, 1990); ocupa una superficie aproximada de $103.86 \mathrm{~km}^{2}$ en los municipios de Tepotzotlán, Teoloyucan, Coyotepec y Huehuetoca, en el estado de México. Es una área formada en el Mioceno superior durante una de las siete fases de vulcanismo que conformaron la Cuenca de México; sus rasgos volcánicos originales apenas se reconocen pues está formada por andesitas muy erosionadas (Mosser, 1975).

La Sierra de Alcaparrosa se caracteriza por poseer un clima templado con verano fresco y largo, con temperatura media anual entre 12 y $18{ }^{\circ} \mathrm{C}$ y una precipitación anual entre 700 y 1000 mm (Núñez, 1990).

De acuerdo con Rzedowski et al. (1964), la Sierra ocupa un intervalo altitudinal entre 2300 y 2750 m; para Nuñez (1990) la altitud oscila entre 2300 y 2990 m y en nuestros trabajos de campo registramos hasta $2930 \mathrm{~m}$. Este intervalo está ocupado por un bosque de Quercus (encinar) - de 2350 a $2990 \mathrm{~m}$ - formado por masas puras o mezcladas de especies de hojas deciduas como $Q$. laeta, Q. rugosa y $Q$. crassipes, entre otras (Núñez, 1990). El matorral de Quercus, constituido por una asociación densa y casi pura de $Q$. microphylla, se desarrolla a 2300-2700 m de altitud. Por su parte, el matorral xerófilo con Opuntia, leguminosas, labiadas y compuestas se distribuye entre 2500-2700 m de altitud. El pastizal se desarrolla entre los 2250 y $2600 \mathrm{~m}$, en áreas con precipitación anual de $600-900 \mathrm{~mm}$; las especies dominantes suelen ser Hilaria cenchroides, Abildgaardia mexicana, Bouteloua radicosa y $B$. hirsuta. Este pastizal parece ser una comunidad clímax (Cruz, 1969). En la práctica es difícil separar los tipos de vegetación ya que los cambios de uno a otro se dan gradualmente (Núñez, 1990).

En el área de estudio se encontró además una asociación riparia con Prunus, Alnus, Salix, Schinus y Crataegus localizada a $2330 \mathrm{~m}$ de altitud que no fue descrita por Núñez (1990).

\section{MATERIAL Y METODO}

Entre 1982 y 1986 se recolectaron 252 ejemplares de musgos en varios tipos de vegetación de la Sierra de Alcaparrosa, según se indica en el Cuadro 1. Los ejemplares determinados se depositaron en MEXU y ENCB y los duplicados se distribuyeron a otros herbarios. Para determinar los elementos fitogeográficos se hizo uso amplio de los datos de distribución de un banco de datos de musgos neotropicales (cf. Delgadillo, 1991).

\section{RESULTADOS Y DISCUSION}

La flora de musgos de la Sierra de Alcaparrosa comprende 84 especies y variedades. En el siguiente listado el nombre cientifico va seguido del número de colecta del 
Cuadro 1. Datos de ejemplares de musgos de la Sierra de Alcaparrosa, Méx. recolectados por A. Cárdenas.

\begin{tabular}{|llll|}
\hline Número & Fecha & $\begin{array}{l}\text { Altitud } \\
\text { en } \mathrm{m}\end{array}$ & Vegetación \\
\hline $1807-1813$ & $13-\mathrm{V}-1982$ & 2330 & Vegetación riparia \\
$1814-1818$ & $13-\mathrm{V}-82$ & 2400 & Encinar abierto caducifolio \\
$1819-1838$ & $13-\mathrm{V}-82$ & $2635-2710$ & Encinar abierto caducifolio \\
$2321-2359$ & $6-\mathrm{XII-82}$ & 2760 & Encinar abierto \\
$2360-2400$ & $7-\mathrm{XII}-82$ & 2420 & Matorral xerófilo \\
$2401-2427$ & $7-\mathrm{XII-82}$ & 2760 & Encinar abierto perturbado \\
$3791-3818$ & $26-\mathrm{VIII}-84$ & $2500-2700$ & Matorral xerófilo \\
$3865-3886$ & $19-1 \mathrm{X}-84$ & 2600 & Encinar \\
$3893-3908$ & $4-\mathrm{XI}-84$ & $2300-2700$ & Pastizal de Hilaria \\
$3909-3912$ & $4-\mathrm{XI}-84$ & $2300-2700$ & Matorral de Quercus \\
$4137-4158$ & $4-\mathrm{V}-85$ & $2890-2930$ & Encinar \\
$4344-4348$ & $19-\mathrm{X}-85$ & 2600 & Pastizal secundario \\
$4503-4505$ & $18-\mathrm{VIII}-86$ & 2450 & Pastizal secundario \\
$4517-4523$ & $24-1 \mathrm{X}-86$ & 2450 & Pastizal secundario \\
$4524-4528$ & $24-1 \mathrm{X}-86$ & 2300 & Lecho de arroyo \\
\hline
\end{tabular}

autor principal y datos del microhabitat; las colecciones de otros individuos se citan por colector y número de colecta. El área de distribución de los elementos fitogeográficos se describe después del listado florístico; el nombre del elemento al que corresponde cada taxon se cita con cada epíteto específico.

Acaulon muticum (Hedw.) C.M. var. rufescens (Jaeg.) Crum. 3797a, 3800a. Suelo húmedo en lugares expuestos. Norteamericano.

Aloina hamulus (C.M.) Broth. 3806, 4524b. Suelo y rocas calcáreas cubiertas por suelo, en lugares expuestos, secos o húmedos. Mesoamericano.

Anacolia laevisphaera (Tayl.) Flow. 2389, 2376a, 2425. Rocas cubiertas por suelo, en lugares sombreados o expuestos y secos. Amplia distribución.

Aonectangium aestivum (Hedw.) Mitt. 2390, 2393, 3807. Rocas cubiertas por suelo, en lugares sombreados o expuestos, secos o húmedos. Amplia distribución.

Anomobryum filiforme (Dicks.) Solms ex Rabenh. 2365; Rzedowski 31267. Roca cubierta por suelo o sobre corteza de Quercus, en lugares sombreados y secos. Amplia distribución.

Anomobryum prostratum C.M. 3810, 3813a. Suelo o rocas, lugares expuestos y mojados. Disyunto.

Archidium alternifolium (Hedw.) Schimp. 4522. Suelo compactado en lugares expuestos y secos. Amplia distribución.

Archidium donnellii Aust. 3811, 4157. Suelo húmedo, en lugares expuestos. Norteamericano. 
Aschisma aethiopicum (Welw. \& Dub.) Lindb. 3865b, 4345, 4521. Suelo compactado en lugares expuestos, secos o mojados. Disyunto.

Barbula orizabensis C.M. 2377. Roca cubierta por suelo, en lugares sombreados y secos. Panamericano.

Barbula spiralis Schimp. ex C.M. 2398, 2406, 3818. Suelo o rocas, en lugares expuestos o sombreados, secos o húmedos. Mesoamericano.

Brachymenium mexicanum Mont. 2338, 2340, 2379b. Rocas secas, en lugares sombreados 0 expuestos. Panamericano.

Brachymenium systylium (C.M.) Jaeg. 1833b, 3794. Rocas o troncos de leguminosas, en lugares sombreados o soleados, secos o húmedos. Amplia distribución.

Brachythecium stereopoma (Spruce ex Mitt.) Jaeg. \& Sauerb. 2371, 4148. Tronco de Opuntia o suelo, en lugares sombreados, húmedos o secos. Panamericano.

Brachythecium tenuinerve Card. 4137b, 2410. Humus o raíz de Quercus, en lugares sombreados, secos o húmedos. Endémico.

Branuia secunda (Hook.) B.S.G. 2333, 2422, 2396. Roca o base de tronco de Quercus, en lugares sombreados o expuestos, secos. Amplia distribución.

Bryoerythrophyllum campylocarpum (C.M.) Crum. 4138, 4149. Suelo, en lugares expuestos o sombreados, húmedos o mojados. Amplia distribución.

Bryoerythrophyllum inaequalifolium (Tayl.) Zand. 3803, 3879, 4527. Suelo húmedo 0 mojado, en lugares soleados o expuestos. Amplia distribución.

Bryoerythrophyllum recurvirostrum (Hedw.) Chen. 1830, 3814. Suelo o rocas, en lugares sombreados o expuestos, húmedos o mojados. Amplia distribución.

Bryum argenteum Hedw. Cruz 1090. Amplia distribución.

Bryum billardieri Schwaegr. 1808, 1823, 2321, 3900. Base de tronco de Quercus o sobre suelo, en lugares sombreados o soleados, húmedos o secos. Amplia distribución.

Bryum chryseum Mitt. 3796, 4347. Suelo húmedo o seco, en lugares sombreados o soleados. Mesoamericano.

Bryum coronatum Schwaegr. 3805, 3813b. Suelo o rocas, lugares expuestos, mojados. Amplia distribución.

Campylium hispidulum (Brid.) Mitt. 2418. Suelo seco en lugares sombreados. Amplia distribución.

Campylopus pilifer Brid. 2383, 3873, 3799. Suelo en lugares expuestos, secos, húmedos o mojados. Amplia distribución.

Ceratodon purpureus (Hedw.) Brid. ssp. stenocarpus (B.S.G.) Dix. 2414. Suelo seco en lugares expuestos. Amplia distribución. Endémico.

Curviramea mexicana (Thér.) Crum. 2394. Roca seca en lugares sombreados. 
Didymodon australasiae (Hook. \& Grev.) Zand. var. australasiae. 1835b, 2395, 3804a. Rocas o suelo, lugares sombreados o expuestos, secos o mojados. Amplia distribución.

Didymodon incrassatolimbatus Card. 2343a, 1836. Tronco de Quercus o rocas, lugares sombreados o soleados, secos. Endémico.

Didymodon revolutus (Card.) Will. 2360, 4524a. Suelo calcáreo, en lugares expuestos y secos. Mesoamericano.

Didymodon rigidulus Hedw. var. gracilis (Schleich. ex Hook. \& Grev.) Zand. 2351, 2362. Suelo seco, en lugares expuestos o soleados. Amplia distribución.

Didymodon rigidulus Hedw. var. icmadophilus (Schimp. ex C.M.) Zand. 1810, 2353, 3884,4151 . Suelo seco o húmedo, en lugares sombreados o expuestos. Amplia distribución.

Didymodon rigidulus Hedw. var. subulatus (Thér. \& Bartr. ex Bartr.) Zand. 3904, 1831,3908 . Suelo o rocas, en lugares soleados o expuestos, secos o húmedos. Disyunto.

Entodon abbreviatus (Besch.) Jaeg. \& Sauerb. 4137a, 4144. Tronco y raíz de Quercus, en lugares sombreados, húmedos o mojados. Endémico.

Entodon beyrichii (Schwaegr.) C.M. 2339, 3910, 4145. Roca o suelo, en lugares expuestos o sombreados, secos, húmedos o mojados. Panamericano.

Entosthodon apiculatopilosus (Card.) Fife. 3808, 3809. Suelo húmedo en lugares expuestos o sombreados. Norteamericano.

Fabronia ciliaris (Brid.) Brid. var. polycarpa (Hook.) Buck. 1813, 2397. Tronco de Alnus, en lugares sombreados y secos. Panamericano.

Fabronia ciliaris (Brid.) Brid. var. wrightii (Sull.) Buck. 2400, 3911, 4147. Base de leguminosa, raíz de Schinus o tronco de Quercus, en lugares sombreados o soleados, secos o húmedos. Panamericano. Disyunto.

Fissidens excurrentinervis Will. 2385, 2404. Suelo seco en lugares expuestos.

Fisșidens milobakeri Koch. 3804c. Suelo mojado en lugares expuestos. Disyunto.

Fissidens repandus Wils. 1811, 2412, 3905, 4143. Suelo o tronco de Quercus, lugares sombreados o expuestos, secos o húmedos. Mesoamericano.

Flowersia campylopus (Schimp. ex C.M.) Griffin \& Buck. 2392. Roca seca en lugares sombreados. Mesoamericano.

Funaria hygrometrica Hedw. var. hygrometrica. Rzedowski 31268. Sobre taludes húmedos. Amplia distribución.

Funaria hygrometrica Hedw. var. calvescens (Schwaegr.) Mont. 2359. Suelo seco en lugares expuestos. Amplia distribución. 
Globulinella globifera (Hampe) Steere. 4525. Suelo calcáreo, en lugares expuestos y secos. Mesoamericano.

Grimmia arizonae Ren. \& Card. 2399. Roca seca en lugares expuestos. Norteamericano.

Grimmia pilifera P. Beauv. 3817. Roca seca en lugares expuestos. Amplia distribución.

Grimmia pulla Card. 2336, 2337, 2401a. Roca seca en lugares sombreados o expuestos. Endémico.

Hedwigia ciliata Hedw. 2342. Roca seca en lugares sombreados. Amplia distribución.

Homomallium mexicanum Card. 2409. Roca seca en lugares expuestos. Norteamericano.

Hygrohypnum sp. 2387. Roca seca en lugares sombreados.

Hyophila involuta (Hook.) Jaeg. \& Sauerb. 2361, 2378, 2381. Suelo o roca, lugares soleados o expuestos, secos. Amplia distribución.

Leptodontium brachyphyllum Broth. \& Thér. 3866. Suelo mojado en lugares expuestos. Amplia distribución.

Leskea angustata Tayl. 2344b, 4142b, 4146b. Tronco de Quercus o tocón, lugares sombreados, húmedos o secos. Disyunto.

Leucodon cf. curvirostris Hampe. 2420. Tronco seco de Quercus, lugares sombreados. Mesoamericano.

Lindbergia mexicana (Besch.) Card. 1818, 3792, 3869b. Tronco de Quercus y roca, lugares sombreados 0 expuestos, húmedos o secos. Mesoamericano.

Micromitrium austinii Aust. 3886a. Suelo compactado en lugares expuestos. Panamericano.

Neckera chlorocaulis C.M. 2415. Tronco seco de Quercus en lugares sombreados. Mesoamericano.

Orthotrichum diaphanum Brid. 1825. Tronco de Quercus, lugares expuestos y secos. Amplia distribución.

Orthotrichum pycnophyllum Schimp. ex C.M. 2331, 2408, 2401b. Tronco de Quercus, rama de arbusto o rocas, lugares expuestos o sombreados, secos. Mesoamericano.

Oxystegus tenuirostris (Hook. \& Tayl.) A.J.E. Smith cf. var. gemmiparus (Schimp.) Zand. 1828. Suelo húmedo en lugares sombreados. Amplia distribución.

Physcomitrium subsphaericum Schimp. 4526. Suelo mojado en lugares expuestos. Mesoamericano. 
Platygyriella pringlei (Card.) Buck. 3869a, 2346, 2358. Tronco y raíz de Quercus - rocas, lugares sombreados, húmedos o secos. Mesoamericano.

Pleuridium aurantiacum Snider \& Delg. 3875b, 4518b. Suelo mojado en lugares expuestos. Endémico.

Pleuridium sullivantii Aust. var. mexicanum (Card.) Cárde. 3801, 3802, 4156b. Suelo húmedo o mojado en lugares expuestos. Endémico.

Pogonatum campylocarpum (C.M.) Mitt. 2426, 2411; Cruz 1100. Suelo seco en lugares sombreados. Panamericano.

Pogonatum oligodus (C.M.) Mitt. 2427, 3872, 4154. Suelo, en lugares expuestos, secos, húmedos o mojados. Amplia distribución.

Pohlia integra (Card.) Shaw. 4528. Suelo mojado en lugares expuestos. Endémico.

Pylaisiella falcata (B.S.G.) Ando. 2330, 2417, 4141. Raíz y tronco de Quercus, lugares sombreados y secos. Amplia distribución.

Racopilum tomentosum (Hedw.) Brid. 2374, 2416. Suelo o tronco de Quercus, lugares sombreados y secos. Amplia distribución.

Rhexophyllum subnigrum (Mitt.) Hilp. 2334. Tronco de Quercus en lugares sombreados y secos. Disyunto.

Rhynchostegium pulchellum (Hedw.) Robins. 2407. Arbusto de Rosaceae, lugares sombreados y secos. Amplia distribución.

Schizymenium serratum (Card. \& Herz. ex Card.) Shaw. 4150. Suelo húmedo en lugares expuestos. Endémico.

Thuidium delicatulum (Hedw.) B.S.G. var. radicans (Kindb.) Crum, Steere \& Anders. 2402. Suelo seco en lugares expuestos. Amplia distribución.

Timmiella anomala (B.S.G.) Limpr. 4140. Suelo húmedo en lugares sombreados. Amplia distribución.

Tortella tortuosa (Hedw.) Limpr. 1829, 2373a. Suelo en lugares sombreados, húmedos o secos. Amplia distribución.

Tortula amphidiacea (C.M.) Broth. 2326b, 2329. Tronco de Quercus, lugaies sombreados y secos. Mesoamericano.

Tortula fragilis Tayl. 1835a, 3870, 3897. Roca, raíz de Quercus o Schinus, lugares soleados o sombreados, secos o húmedos. Amplia distribución.

Tortula obtusissima (C.M.) Mitt. 3901, 3902, 3883. Suelo en base de leguminosa o sobre rocas, lugares soleados, sombreados o expuestos, húmedos o mojados. Disyunto.

Tortula papillosa Wils. 3815b. Tronco de Quercus, lugares sombreados y húmedos. Amplia distribución. 
Trichostomum brachydontium Bruch ex F. Müll. 2376b, 2349, 2352. Roca cubierta por suelo, en lugares sombreados o expuestos, secos. Amplia distribución.

Uleobryum curtisii I. Stone. 3865a. Suelo mojado en lugares expuestos. Disyunto.

Weissia subangustifolia (Thér.) Zand. 3867, 3880. Suelo húmedo en lugares expuestos. Endémico.

Zygodon ehrenbergii C.M. 2326a. Tronco de Quercus en lugares sombreados y secos. Mesoamericano.

Estos taxa se distribuyen en casi todos los tipos de vegetación del área y a todo lo largo del perfil altitudinal de la Sierra. Aunque pueden existir varias explicaciones, tal vez la baja humedad atmosférica y la concentración de la lluvia (75 a $85 \%$ ) en los meses de mayo a septiembre, con una prolongada época seca de 5 a 7 meses (Rzedowski et al., 1964), hacen que los musgos mejor representados sean los de la familia Pottiaceae que poseen adaptaciones a condiciones de sequía. Los musgos cleistocárpicos (Acaulon, Archidium, Aschisma, Pleuridium y Uleobryum), cuyo ciclo de vida es efímero, también están bien representados y ofrecen una estrategia adaptativa distinta al clima de la región. La presencia y diseminación de estas plantas parece ser favorecida por el disturbio al que se ha sometido al área por muchos años.

En comparación con la Sierra de Pachuca, también situada en la mitad septentrional del Valle de México y con una flora conocida de 169 especies y variedades de musgos, la Sierra de Alcaparrosa es florísticamente más pobre. La diferencia puede deberse a que la Sierra de Pachuca tiene una vegetación arbórea más diversa (que incluye bosques de Abies, Abies-Quercus y Juniperus); a su fluctuación en la cantidad y forma de la precipitación (la precipitación anual oscila entre 600 y $1500 \mathrm{~mm}$, con niebla y rocío frecuentes); y a un intervalo altitudinal mayor, de 2400 a $3050 \mathrm{~m}$ (Alfaro y Castillo, 1986). Es interesante señalar, sin embargo, que el número total de plantas vasculares en la Sierra de Alcaparrosa es de 868 taxa mientras que en la vertiente sur de la Sierra de Pachuca sólo se han registrado alrededor de 800 especies (Medina, 1980; Núnez, 1990).

Desde el punto de vista fitogeográfico es importante señalar la presencia en la Sierra de Alcaparrosa de un contingente (siete especies) de musgos cleistocárpicos; no se ha observado un número semejante en sitios de tamaño similar en México. Se ha sugerido (Delgadillo, 1985; 1987a) que el Eje Neovolcánico ha actuado como barrera y vía de migración de musgos y que algunas especies, entre ellas las de musgos cleistocárpicos, han podido cruzarlo en ambas direcciones, por lo que ahora se distribuyen en los valles al norte y al sur de ese macizo. Este hecho es particularmente notable porque los musgos cleistocárpicos no parecen tener mecanismos de diseminación efectiva que hayan promovido su distribución entre la Cuenca de México y los valles del suroeste de Puebla en donde los hemos observado. Los musgos cleistocárpicos de la Sierra de Alcaparrosa son también de interés porque algunas especies como Aschisma aethiopicum y Uleobryum curtisii tienen patrones de distribución disyuntos que ligan a esta flora con la de sitios muy alejados, como Africa y Australia en los dos ejemplos precedentes.

Por su distribución, los musgos de la Sierra de Alcaparrosa pueden separarse en seis elementos fitogeográficos (Cuadro 2). El elemento endémico está representado por 10 especies sólo conocidas de México; el elemento norteamericano comprende cinco 
especies distribuidas desde el sur y sureste de los Estados Unidos de América hasta el centro de México. Exceptuando a Barbula orizabensis y Brachymenium mexicanum, las especies del elemento panamericano están representadas en las principales divisiones del Continente Americano.

Cuadro 2. Elementos fitogeográficos en la flora de musgos de la Sierra de Alcaparrosa.

\begin{tabular}{|lcr|}
\hline Elemento & No. especies & $\%$ \\
\hline Endémico & 10 & 12.0 \\
Norteamericano & 5 & 6.0 \\
Panamericano & 8 & 10.0 \\
Disyunto & 9 & 11.0 \\
Mesoamericano & 15 & 18.0 \\
Amplia distribución & 36 & 43.0 \\
\hline
\end{tabular}

Además de las dos disyunciones intercontinentales a que hicimos referencia en un párrafo anterior, el elemento disyunto incluye siete especies con distribución discontinua entre México y Sudamérica. Por su parte, el elemento mesoamericano, con quince especies, tiene una distribución que se extiende esencialmente desde México hasta el norte de Sudamérica (cf. Delgadillo, 1987b); entre ellas, Aloina hamulus, Globulinella globifera y Platygyriella pringlei, sólo se encuentran en el extremo norte del área de distribución, desde México hasta Honduras o El Salvador. Por último, el contingente más numeroso es el constituido por 36 especies ampliamente distribuidas en el mundo.

La flora de musgos de la Sierra de Alcaparrosa es comparativamente pequeña, pero incluye los elementos fitogeográficos fundamentales de la flora mexicana de altas elevaciones. Su rasgo sobresaliente es la ausencia casi completa de taxa de filiación tropical y la abundancia de especies de amplia distribución. El significado de estos patrones de distribución ha sido abordado en otras publicaciones (Delgadillo, 1971; 1987a \& b; 1988) y será reevaluado al concluir los trabajos florísticos para el Valle de México y para el Eje Neovolcánico.

\section{AGRADECIMIENTOS}

Agradecemos al Dr. Jerzy Rzedowski la revisión del manuscrito y a Ma. Bernardina Bello su apoyo en la obtención de datos de herbario. El mapa de la zona de estudio fue preparado por el Sr. Felipe Villegas.

\section{LITERATURA CITADA}

Alfaro O., A.P. y X.J. Castillo D. 1986. Distribución por tipos de vegetación de los musgos de la Sierra de Pachuca, Hidalgo. Tesis Profesional. ENEP-Zaragoza, Universidad Nacional Autónoma de México. México, D.F. 55 pp.

Cárdenas S., A. 1987. Notas sobre la variación fenotipica y taxonomía de Pleuridium Brid. (Musci) en el Valle de México. Cryptogamie, Bryol. Lichénol. 8: 305-310.

Cárdenas S., A. 1988. Los musgos cleistocárpicos del Valle de México, México. Bryologist 91: 214-216. 
Cárdenas S., A. 1989. Nuevos registros para la flora de musgos de México y del Valle de México. Anales Inst. Biol. UNAM, Ser. Bot. 58: 93-95.

Crum, H.A. 1951. The Appalachian-Ozarkian element in the moss flora of Mexico with a check-list of all known Mexican mosses. Ph.D. Dissertation. Univ. Michigan. Ann Arbor. 504 pp.

Cruz C., R. 1969. Contribución al conocimiento de la ecología de los pastizales en el Valle de México. Tesis Profesional. Escuela Nacional de Ciencias Biológicas, Instituto Politécnico Nacional. México, D.F. 235 pp.

Delgadillo M., C. 1971. Phytogeographic studies on alpine mosses of Mexico. Bryologist 74: 331-346.

Delgadillo M., C. 1985. The Neovolcanic Belt of Mexico as a barrier and route of migration for mosses. Monogr. Syst. Bot. Missouri Bot. Gard. 11: 41-44.

Delgadillo M., C. 1987a. Moss distribution and the phytogeographical significance of the Neovolcanic Belt of Mexico. Journ. Biogeogr. 14: 69-78.

Delgadillo M., C. 1987b. The Meso-American element in the moss flora of Mexico. Lindbergia 12: 121124.

Delgadillo M., C. 1988. Floristic corridors for moss distribution across the Neovolcanic Belt of Mexico. I.The Tuxpan corridor. Journ. Bryol. 15: 165-175.

Delgadillo M., C. 1991. Los patrones de distribución de los musgos neotropicales. In: Delgadillo M., C., Memoria, II Simposio Latinoamericano de Briología. Sociedad Latinoamericana de Briología \& Instituto de Biología. México, D.F. pp. 39-48.

Medina C., M. 1980. Análisis fitogeográfico de la vertiente sur de la Sierra de Pachuca, Estado de Hidalgo. Tesis Profesional. Escuela Nacional de Ciencias Biológicas, Instituto Politécnico Nacional. México, D.F. $58 \mathrm{pp}$.

Mosser, F. 1975. Historia geológica de la Cuenca de México. In: Memoria de las obras del Sistema de Drenaje Profundo del Distrito Federal. Departamento del Distrito Federal, México, D.F. Vol. 1. pp. 7-38.

Núñez R., J. E. 1990. Estudio florístico de la vertiente oriental de la Sierra de Alcaparrosa, en el Estado de México. Tesis Profesional. ENEP-Iztacala, Universidad Nacional Autónoma de México. México, D.F. 122 pp.

Rzedowski, J., G. Guzmán, A. Hernández C. y R. Muñíz. 1964. Cartografía de los principales tipos de vegetación de la mitad septentrional del Valle de México. An. Esc. Nac. Cienc. Biol. Méx. 13: 3157. 